\title{
Molecular-Docking Study of Anti-Stress Natural Compounds Against GABAa Receptor Portends the Novel Approach to Stress Treatment
}

\author{
Abhishek Negi, Parul Singh, Nancy Taneja, Shalini Mani* \\ Department of Biotechnology, Jaypee Institute of Information Technology, Sector 62, Noida, India.
}

\author{
ARTICLE INFO \\ Article history: \\ Received on: 05/05/2017 \\ Accepted on: 05/02/2018 \\ Available online: 30/12/2018
}

\section{Key words:}

Stress, Lorazepam, GABAa

receptor, docking.

\begin{abstract}
Introduction: During stress our body responds by activating the nervous system and hormones. The stress response is conveyed by hypothalamic-pituitary-adrenal axis and is responsible for neuroendocrine adaptation of stress response. Hypothalamic-pituitary-adrenal activity is governed by corticotrophin releasing hormone neurons. Increased production of corticotrophin releasing hormone is associated with various stress related metabolic and neurological disorders. The activity of CRH neurons is largely regulated by Gamma-aminobutyric acid (GABA) and is controlled by GABAergic inhibition. To lower down the effect of stress, various synthetic drugs are used which target GABAa receptor and activates GABAa activity, producing calming effects but also comes along with various side effects. Hence anti-stress herbs is used as an alternative therapy which helps improving the adrenal system has no side effects and are safe for long term use. Method: Our current study is an attempt to analyze the possible mechanism of action of some compounds commonly present in extracts of different anti-stress herbs by using AutoDockVina. Result: As a result of our docking studies with GABAa protein, we have observed that some herbal compounds such as Rosmarinic Acid and Kaempferol are showing comparable binding affinity towards GABAa receptor, like Lorazepam. Few other compounds sharing common binding site like Lorazepam, indicates that their mechanism of action may also be through activating GABAa receptor.
\end{abstract}

\section{INTRODUCTION}

Definition of stress is not clear but often described as feeling of being anxious or worried when an individual perceives surrounding demands. Stress has a very wide spectrum of effect on different parts of body in different ways and thus can affect various organs, thoughts, feelings and behavior. If stress is left unchecked, it may ultimately lead to various mental and physical illnesses such as headaches, upset stomach, elevated blood pressure, chest pain, heart disease (Romero et al., 2007).

The hypothalamus in the brain is the in-charge of different stress responses. When a stress response is triggered, hypothalamus sends signals to pituitary gland and the adrenal medulla. The activated hypothalamus stimulates the pituitary gland to produce adrenocorticotropic hormone. It travel through

${ }^{*}$ Corresponding Author

Shalini Mani; Senior Assitant Professor, Department of Biotechnology,

Jaypee Institute of Information Technology, Sector 62, Noida.

E-mail: shalini.mani@jiit.ac.in; mani.shalini@gmail.com blood stream to the adrenal cortex and stimulates the adrenal gland to produce the hormone corticosteroid, including cortisol. The cortisol is a stress hormone which enables the body to maintain steady supplies of blood sugar. Adequate and steady blood sugar levels help person to cope with prolonged stressor, and helps body to return to normal (Glover et al., 2010). These stress responses are governed by corticotrophin releasing hormone neurons. The activity of these neurons is largely controlled by robust Gammaaminobutyric acid (GABA) mediated inhibition. GABA is the most widely distributed inhibitory neurotransmitter in the central nervous system. GABA influences our mood because it reduces high levels of the hormones adrenalin, noradrenalin and dopamine.

Upon neuronal activation, GABA is released from vesicles into the synapse, where it can act on postsynaptic receptors, or diffuse into the extracellular space and activate extra synaptic receptors on postsynaptic neurons (Kalueff et al., 1996; Roozendaal et al., 1997; Nutt et al., 2006). GABA is then removed from the extracellular space by GABA transporters located on presynaptic neurons and glia that take up GABA into cells away 
from postsynaptic GABA receptors (Luján et al., 2005; Mihic et al., 1997). Extracellular levels of GABA are regulated by the balance between GABA release from presynaptic vesicles and GABA uptake by GABA transporters (Sahley et al., 2010). As such, GABA limits the excitability of neuronal activity in all areas of the brain. GABA inhibits the cells from firing, diminishing the anxiety related messages from reaching the cortex (Glover et al., 2010). All the functions of GABA is mediated through it specific receptor; GABA receptor. There are two main types of GABA receptors: fast-acting ionotropic GABAa and slow acting ionotropic GABAc (Mihic et al., 1997).

To lower down the effect of stress, different sedative medications are commonly recommended by clinicians. These sedatives depress the activity of the central nervous system (causing a sense of relaxation, reduced anxiety and tension, sleepiness, and slowed breathing. One of the most commonly prescribed groups of sedatives is the Benzodiazepines which include alprazolam (Xanax), clonazepam (Klonopin), diazepam (Valium), lorazepam (Ativan), triazolam (Halcion), temazepam (Restoril), and chlordiazepoxide (Librium). Benzodiazepines and alcohol produce their calming effects by activating GABA receptor which ultimately inhibit brain activity and thus slow and calm down the body (Kulkarni et al., 1996). However, the use of these drugs for long period is known to exhibit some severe side effects at physiological and psychological level such as slurred speech, impaired ability to walk around, poor judgment, slowed reflexes, low blood pressure, sexual dysfunction, memory loss. Hence, there is always a need of alternative therapy for stress management (Kinnersley et al., 2000). As per published literature, a large number of herbs are known to help improving the function of adrenal system and reduces the stress too. Additionally, these anti-stress herbs are safe for long term use (Pawar et al., 2012).

Various epidemiological studies have shown that a diet rich in plant derived bioactive dietary constituents has protective effect against stress in human. Many herbs have their own specific components but few compounds are still common in these herbs. Kaempferol, Rosmarinic acid, Caffeicacid and Eugenol are few of the common phytocompounds used in many traditional medicines for stress management (Fujisawa and Murakami, 2016; Lakshmi et al., 2011; Lee et al., 2014; Friedman et al., 2015). These compounds are predominantly present in different well studied antistress herbs such as Sarcandraglabra, Curcuma longa and Perillaeherba etc. (Table 1). Numerous preclinical studies have shown there wide range of pharmacological activities and their safety in usage (Calderon et al., 2011; Vladimir et al., 2014; Khalil et al., 2017).

Table 1: Common anti-stress herbs and their important compounds.

\begin{tabular}{|c|c|c|c|c|c|}
\hline Anti-stress Herbs & Genus & Family & Common Name & Important compounds & Reference \\
\hline Oryzasativa & Oryza & Poaceae & Rice bran & $\gamma$-oryzanol, tocopherol, tocotrienols. & Heinemann et al. (2008) \\
\hline Ginkgo biloba & Ginkgo & Ginkgoaceae & Maidenhair tree & Kaempferol, terpene lactones, ginkgolide, tocopherols & $\begin{array}{l}\text { Zahradníková et al. } \\
\qquad \text { (2007) }\end{array}$ \\
\hline Panax ginseng & Panax & Araliaceae & Ginseng (Asian) & Triterpenoid, ginsensoid, panaxtriol, germanium & Heffern (1976) \\
\hline Eugenia carophyllus & Syzygium & Myrtaceae & Clove & Eugenol, carophylleneoxide, eugenylaceteate, & Uddin et al. (2017) \\
\hline Rhodiolarosea & Rhodiola & Crassulaceae & Golden root & Rosarin, rodiolin, tyrosol, triterpenes & Colitti (2014) \\
\hline Curcuma longa & Curcuma & Zingiberaceae & Turmeric & Cinnamic acid, eugenol, caffeic acid, capsacin, gingerol & Gupta et al. (2010) \\
\hline Perillaeherba & Perilla & Lamiaceae & Perilla & Rosmarinicacid, caffeic acid, kaempferol & Ito et al. (2008) \\
\hline Sarcandraglabra & Sarcandra & Chloranthaceae & Gumok & $\begin{array}{l}\text { 5-Caffeoylquinic acid, 3-caffeoylquinic acid, caffeic acid, } \\
\text { 4-O-b-D-glycopyranosyl rosmarinic acid }\end{array}$ & He et al. (2009) \\
\hline Withaniasomnifera & Withania & Solanaceae & Indian ginseng & Withaferin-a, Sitoindoside - IX-H, Sitoindoside - X-Palmitoyl & $\begin{array}{l}\text { (Kaur et al., 2001; } \\
\text { Geetha et al., 2008) }\end{array}$ \\
\hline Ficusbenghalensis & Ficus & Moraceae & Bargad & Rutin and quercetin & Lotankar et al. (2016) \\
\hline
\end{tabular}

The presence of these common components in many of these anti-stress herbs indicates towards their most probable role in managing the stress. But the mechanism of action of these compounds as possible anti-stress factor is not yet fully studied. It will be interesting to highlight the possible target and further propose the mechanism of action of these anti-stress herbs. Hence in order to understand it, our study was conducted further by taking the well-studied synthetic drug molecule, as a positive control. As most of the anti-stress synthetic drug targets and activates GABAa receptor protein, hence in our study, we also docked the common anti-stress herbal components such as Kaempferol, Rosmarinic acid, Caffeic acid and Eugenol with GABAa receptor and compared the docking results with that of a common synthetic drug Lorazepam.

\section{MATERIAL AND METHODS}

In order to evaluate the affinity of different anti-stress herbal components towards GABAa, mainly four compounds namely, Eugenol (ID 3314), Rosmarinic-acid (ID 5281792), Kaempferol (ID 5280863) and Caffeic-acid (ID 689043) were selected. Lorazepam (ID 3958) a synthetic drug molecule was also used as positive control for the docking study.

\section{Protein sequence homology search}

BLAST (Basic Local Alignment Search Tool) is an algorithm for comparing primary biological sequence information, such as the amino-acid sequences of different proteins or the nucleotides of DNA sequences (Kloet et al., 2003). GABAa receptor sequence of Homo sapiens (ID 119619829) was run on pBLAST against non-redundant protein database with default parameters. The sequences of the target protein and those of the orthologs found were extracted from the NCBI Protein database.

\section{Evolutionary analysis of sequence}

For evolutionary analysis of each amino acid, the FASTA format of the GABAa protein sequences from all the above 
selected organisms was used under the option of Estimate Position By Position rates in MEGA software version 7 (Pennsylvania State University, License-Proprietary freeware) (Kumar et al., 2016). Mean (relative) evolutionary rate were shown for each site next to the site number. These rates are scaled such that the average evolutionary rate across all sites is 1 . This means that sites showing a rate $<1$ are evolving slower than average, and those with a rate $>1$ are evolving faster than average. These relative rate were estimated under the Jones-Taylor-Thornton model $(+\mathrm{G})$ (Jones et al., 1992). A discrete Gamma (+G) distribution was used to model evolutionary rate differences among sites ( 5 categories). All positions containing gaps and missing data were eliminated. There were a total of 491 positions in the final dataset.

\section{Sequence aignificance}

As domains play an influential role in deciding protein structure and function. Hence, in order to identify the domains, GABAa sequence was run on Interpro, online software of EMBL-EBI.

\section{Computational docking}

In order to evaluate the affinity of different ligands (synthetic as well as different components of anti-stress herbs) towards Gamma-Aminobutyric Acid Receptor A (GABAa), computational docking was performed using Autodockvina 1.1.2 (Trott and Olson, 2010; Seeliger and Groot, 2010). The files for all these ligands were downloaded from NCBI-PubChem. As the GABAa receptor protein is active in homopentamer state, not in monomeric form, hence, the crystal structure of human GABAaRbeta3 homopentamer was downloaded from PDB database (PDB ID 4COF) (Figure 1). Active site prediction was done using published literature (Miller et al., 2014) and Uniprot. Before docking, protein structure was prepared by adding hydrogen and removing water molecules.

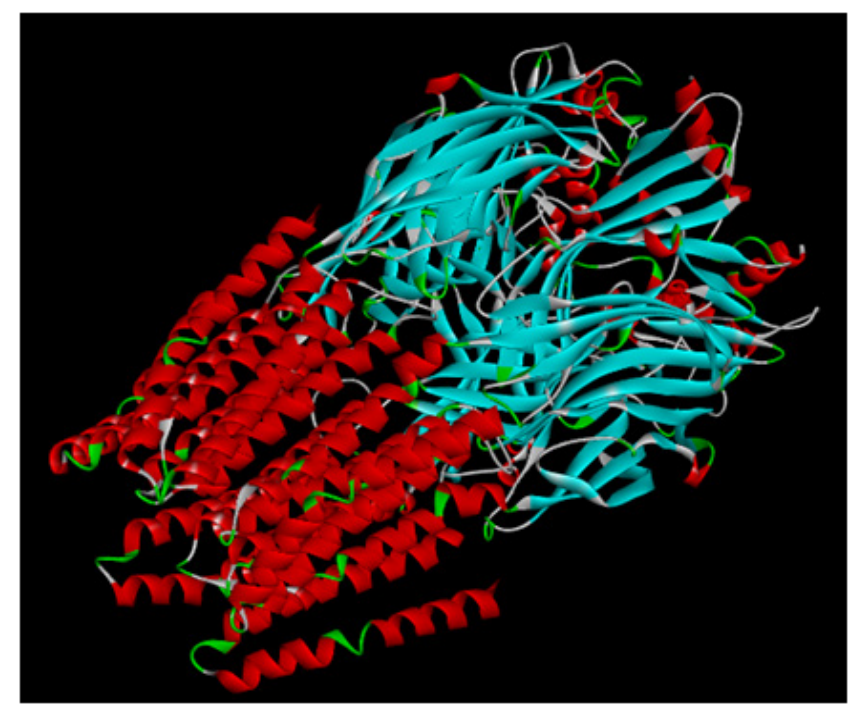

Fig. 1: Crystal structure of human GABAaR-beta3 homopentamer obtained from PDB database (PDB ID 4COF).

\section{Structural Visualization}

For visualizing the result of docking Schrödinger PyMOL v1.8.6.0, molecular visualization software (Seeliger and Groot, 2010) that can produce high-quality 3D images of small molecules and biological macromolecules was used.

\section{RESULT AND DISCUSSION}

In order to find close relative of the query human GABAa protein, pBLAST was run and from the similarity search result, primates with at least $98 \%$ similarity and high E value (that is zero), 7 closest relatives namely Pan paniscus, Pongoabelii, Macacanemestrina, Cebuscapucinus imitator, Saimiriboliviensis, Propithecuscoquereli and Callithrixjacchus were selected. For the purpose of studying the evolutionary rates of the protein on site by site basis, sequences of all the selected organisms were ran on MEGA software. As a result, across the entire length of GABAa protein sequence (493 amino acids), total 5 separate gamma parameters (between 0.1 to 1.0 ) were suggested, for each amino acid (AA) residue. Taking the highest gamma parameters for each site, a graph was prepared and on comparison with average gamma value (0.206), those AA sites were identified which had, highest and lowest rates of evolution. As a result the AAs stretch from 24 to 384 was found to be below the average value and so comparatively conserved (Figure 2). Similarly, Interpro search showed 2 domain that lie in the conserved region of the protein i.e. 68-277 (Neurotransmitter-gated ion-channel ligand-binding domain (IPR006202)) and 276-481 (Neurotransmitter-gated ion channel transmembrane-domain (IPR006029)). So any ligand binding in these conserved regions could be considered a better ligand since chances of mutation would be comparatively less in this region.

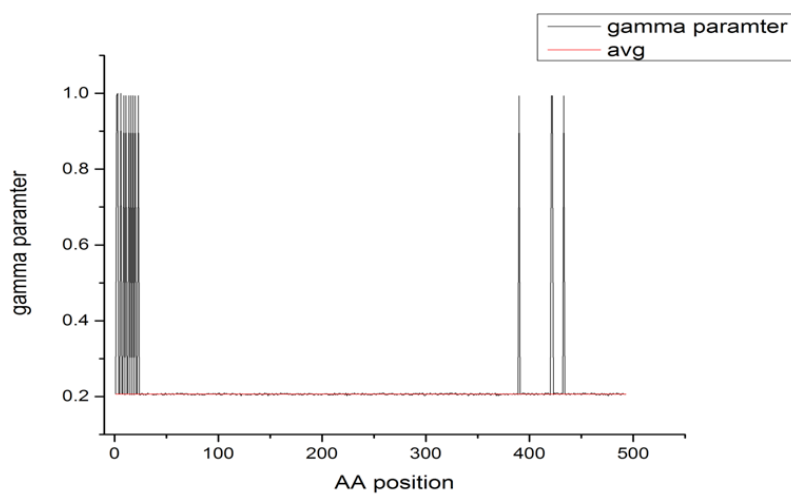

Fig. 2: Graph showing the gamma parameter for each amino acid of GABAa receptor. Average Gamma parameter is 0.206 and showing AA positions that have lower and higher evolutionary rate in the protein sequence.

Further, in-silico docking was done to produce many conformation of protein-ligand complex, among which at least one native-like conformation was selected and its binding affinity was checked. Docking is important to determine the properties associated with protein-ligand interaction such as binding energy and hydrophobicity as well. Binding affinity of all the selected natural compounds was compared with that of Lorazepam drug.

In our analysis, all those natural compounds which exhibited almost similar binding affinity like Lorazepam, was suggested to bind to GABAa receptor. Further the conformation 
with highest binding affinity was considered to be the most suitable docking pose. As shown in Table 2, docking score of all ligand was found ranging between -6.9 to $-9.2 \mathrm{kcal} / \mathrm{mol}$. It was observed that out of all the natural compounds, Kaempferol and Rosmarinic acid showed the binding affinity close to Lorazepam ( -9.2 and $-8.8 \mathrm{kcal} / \mathrm{mol}$ respectively), however the other two compounds like Eugenol and Caffeic acid showed relatively low affinity towards the GABAa receptor.

Table 2: Comparative docking study of the different ligands to the target (GABAa receptor protein).

\begin{tabular}{ccc}
\hline Compound & Affinity (Kcal/mol) & Position \\
\hline Lorazepam & -9.1 & TYR (157,205), THR (202) \\
Kaempferol & -9.2 & SER (156) \\
& -8.8 & THR (176), ASN (41), GLU (155), TYR \\
Rosmarinic acid & -6.9 & GLN (64), THR (202), TYR (205) \\
Eugenol & -7.2 & TYR (62), ASP (43), ARG (180), GLN \\
Caffeic-acid & & (64), THR (202), TYR (205) \\
\hline
\end{tabular}

Further binding position and number of bonds of these ligand-protein complexes were checked using PyMol. The GABAa protein is 493 AAs long and with a binding affinity of $-9.1 \mathrm{kcal} /$ mol, Lorazepam form 3 hydrogen bonds inside the ligand binding domain of receptor protein at TYR $(157,205)$ and THR (202) positions (Table 2). It binds with hydroxyl group present in the drug structure as shown in Figure 3a.

Kaempferol also bind to GABAa protein with almost similar affinity $(-9.2 \mathrm{kcal} / \mathrm{mol})$ like Lorazepam. It binds to the receptor protein by one hydrogen bond at SER (156) (Figure $3 b)$. Though this position is different from the position where Lorazepam binds to the receptor, however the SER at 156 position is also located in the conserved ligand binding domain of GABAa receptor. This may be the reason that the binding affinity of Kaempferol was comparable to that of Lorazepam. Further, it is known that Kaempferol is a flavanoid having a prenyl group that might interact with human GABAa protein through additional hydrophobic and anionic characteristic moieties (Jung et al., 2012). The presence of a 4'-hydroxyl group on the Kaempferol can also increase the affinity and inhibitory potency against GABAa via interaction with SER (156) at the active site.

Next to Kaempferol, Rosmarinic acid was another important compound in our study. It was found that Rosmarinic acid with binding affinity of $-8.8 \mathrm{kcal} / \mathrm{mol}$, formed 5 Hydrogenbond with the GABAa receptor and AA residue conscientious for its binding interactions were THR (176), ASN (41), GLU (155), TYR (97) and ARG (180) (Figure 3c). Out of all these five AAs, except ASN (41), rest all the AAs were present in conserved ligand binding domain of GABAa receptor protein. Since Rosmarinic acid has only one double bond between the two aromatic rings, it has a flexible structure (Dileep et al., 2011), which may help in binding to different positions of the receptor protein. Further there are eight oxygen atoms which can act as hydrogen bond acceptors/donors in rosmarinic acid important for interaction with other proteins.

While comparing the binding positions of Kaempferol and Rosmarinic acid, it is interesting to note that, in case of Kaempferol the bond was formed in the ligand binding domain while as for Rosmarinic acid one bond ASN (41) was not formed inside the conserved ligand binding domain region of the GABAa protein which was further reflected in terms of low binding affinity for Rosmarinic acid.

Next to Kaempferol and Rosmarinic acid, Caffeic acid showed the binding affinity of $-7.2 \mathrm{kcal} / \mathrm{mol}$. It was found to form 6 Hydrogen-bond with receptor protein at TYR (62), ASP (43), ARG (180), GLN (64), THR (202), TYR (205) AA residues (Figure $3 \mathrm{~d}$ ). Out of all the 6 positions, only three AA reside in ligand binding domain of receptor, remaining three (TYR (62), ASP (43) and GLN (64)) was not the part of conserved binding domain. This may be the reason that the overall binding affinity for Caffeic acid was less than Lorazepam, Kaempferol and Rosmarinic acid as well. Similar to Caffeic acid, Eugenol also showed lower binding affinity $(-6.9 \mathrm{kcal} / \mathrm{mol})$ towards GABAa receptor protein. It formed 3 Hydrogen-bond at GLN (64), THR (202), TYR (205) (Figure 3e) and thus have common binding partner like Caffeic acid. All three compounds, Lorazepam, Eugenol and Caffeic acid interact with the GABAa receptor protein at two common position that is THR (202), TYR (205). However there was significant difference in the binding affinity of these three ligands. It may be due to the structural differences amongst them.

So after analyzing all the results of our selected ligands, the study suggested that Rosmarinic acid and Kaempferol (with a binding affinity of -8.8 and $-9.2 \mathrm{kcal} / \mathrm{mol}$ respectively) binds at conserved ligand binding domain of GABA receptor and could be use as substitute for Lorazepam drug. While the other two ligands, Eugenol and Caffeic-acid showed relatively low binding affinity towards the protein receptor and were not considered further.

\section{CONCLUSION}

The protein-ligand interaction plays a significant role in drug design. Our study shows that all the studied herbal compounds are binding to GABAa receptor at its conserved sequence region from 24 to 384 and at Neurotransmitter-gated ion-channel ligand-binding domain. Additionally, the binding affinity of Rosmarinic Acid and Kaempferol (-8.8 and $-9.2 \mathrm{kcal} /$ mol, respectively) with receptor GABAa is highly comparable to the binding affinity of drug Lorazepam $(-9.1 \mathrm{kcal} / \mathrm{mol})$. So, it can be proposed that extracts of some herbs such as Sarcandraglabra, Perillaeherba, Ginkgobiloba are almost equally good anti-stress factors as Lorazepam and can be used as a substitute for the synthetic drug. However, a complete analysis of these compounds for their absorption, distribution, metabolism, excretion/toxicity properties is need to be studied further by different in-vitro and in-vivo assays.

\section{ACKNOWLEDGEMENT}

Authors acknowledge Jaypee Institute of Information Technology, Noida for providing all the supports, to conduct this study.

\section{CONFLICT OF INTEREST}

There are no conflicts of interests. 
a)

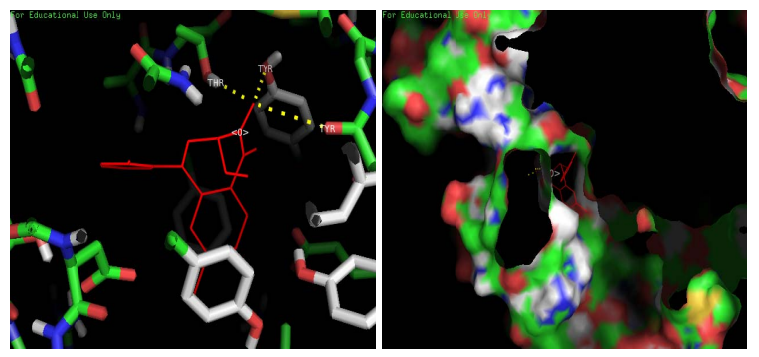

b)

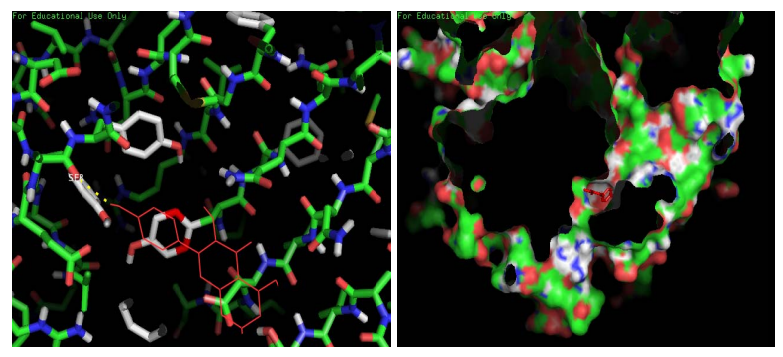

c)

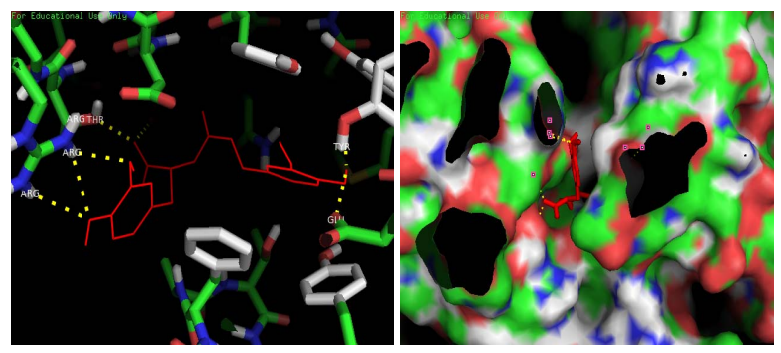

d)
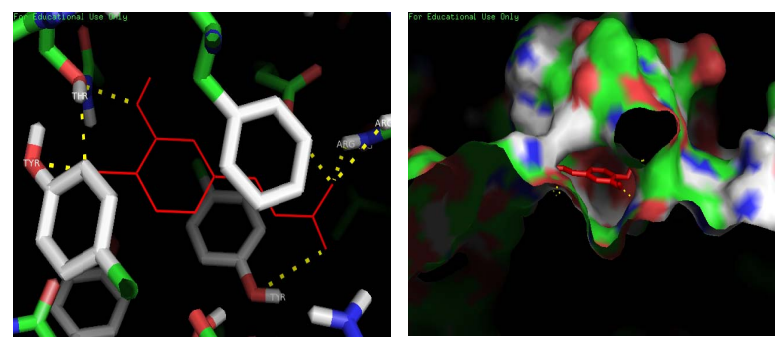

e)
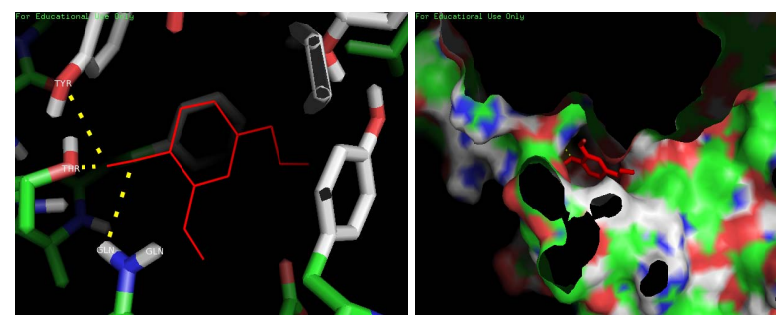

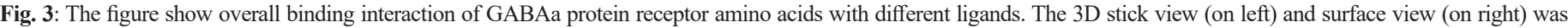

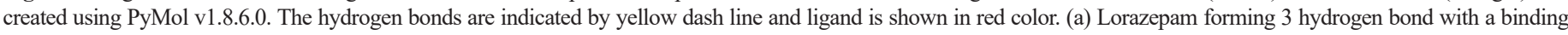

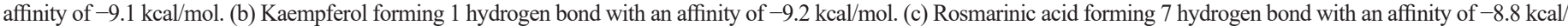
mol. (d) Caffeic acid forming 6 hydrogen bond with a binding affinity of $-7.2 \mathrm{kcal} / \mathrm{mol}$. (e) Eugenol forming 3 hydrogen bond with a binding affinity of $-6.9 \mathrm{kcal} / \mathrm{mol}$. 


\section{REFERENCES}

Altschul SF, Gish W, Miller W, Myers EW, Lipman DJ. Basic local alignment search tool. J Mol Biol., 1990; 215:403-10.

Calderon-Montano JM, Burgos-Moron E, Perez-Guerrero C, and Lopez-Lazaro M. A Review on the Dietary Flavonoid Kaempferol. Mini Rev Med Chem., 2011;11:298-344.

Colitti M. Rhodiolarosea: From the Adaptogenic Role to the Anti-Adipogenic Effect?, Endocrinol Metab Synd., 2014; 3:3.

De Kloet E R. Hormones, brain and stress. Endocr. Regul., 2003; 37: $51-68$.

Desai SK, Desai SM, Navdeep S, Arya P, and Pooja T. Antistress activity of Boerhaaviadiffusa root extract and a polyherbal formulation containing Boerhaaviadiffusa using cold restraint stress model. Int. J. Pharm. Pharm. Sci., 2011; 3: 130-132.

Dileep KV, Tintu I, Sadasivan C. Molecular Docking Studies of Curcumin Analogs with Phospholipase A2. InterdiscipSciComput Life Sci., 2011, 3: 189-197.

Fujisawa S, Murakami Y. Eugenol and Its Role in Chronic Diseases. AdvExp Med Biol., 2016;929:45-66.

Geetha KM and Murugan V. Antistress activity of a herbal extract. Acta Horticulturae., 2008; 786:99-10.

Glover V, Connor TGO and Donnell KO. Prenatal stress and the programming of the HPA axis. Neurosci Biobehav Rev., 2010;35:17-22.

Gupta SK, Lather A, Jaiswal V, Garg S, Jyoti, Kumar A Phytochemistry of Curcuma Longa - An Overview. J Pharm Biomed Sci., 2010;48: 29512959

He R, Yao X, Li H, Dai Y, Duan Y, Li Y, and Kurihara H. The Anti-stress Effects of Sarcandraglabra Extract on Restraint-Evoked Immunocompromise. Biol Pharm Bull., 2009; 32:247-252.

Heffern R. Complete Book of Ginseng: by Heffern. 1976:98.

Heinemann R, Xu Z, Godber J, and Lanfer-Marquez U. Tocopherols, Tocotrienols, and $\gamma$-Oryzanol Contents in Japonica and Indica Subspecies of Rice (Oryza sativa L.) Cultivated in Brazil. Cereal Chem, 2008; 85: 243-247.

Ito N, Yabe T, Gamo Y, Nagai T, Oikawa T, Yamada H, Hanawa T. Rosmarinic acid from PerillaeHerba produces an antidepressant-like effect in mice through cell proliferation in the hippocampus. Biol Pharm Bull., 2008;1:1376-1380.

Jones DT, Taylor WR, Thornton JM. The rapid generation of mutation data matrices from protein sequences. Computer Appl Biosci., 1992; 8: 275-282.

Jung H, Moon H, Oh S, Kim B, Sohn H, and Choi J. Kinetics and molecular docking studies of kaempferol and its prenylated derivatives as aldose reductase inhibitors. Chem. Biol. Interact., 2012;197:110-118.

Kalam S, Yegnambatla R, Periasamy G, Kasarla S, and Yasmeen N. Antioxidant and Anti-inflammatory Activities of Flowers of Plumeriarubra L. f. rubra and Plumeriarubra f. lutea: A Comparative Study. Res J Pharma, Biol and Chem Sci., 2013; 4: 743-756.

Kalueff A, Nutt DJ. Role of GABA in memory and anxiety. Dep and Anx.1996;4:100-10.

Kaur P, Mathur S, Sharma M, Tiwari M, Srivastava K, and Chandra R. A biologically active constituent of withaniasomnifera (ashwagandha) with antistress activity. Indian J Clin Biochem., 2001;16:195-198.

Khalil A, Rahman U, Khan M, Sahar A, Mehmood T and Khan M, Essential oil Eugenol: sources, extraction techniques and nutraceutical perspectives" RSC ADV., 2017; 7, 52: 32669-32681.

Kinnersley AM and Turano FJ. Gamma Aminobutyric Acid (GABA) and Plant Responses to Stress. CRC. Crit Rev Plant Sci., 2000;19: 479-509.

Kulkarni SK and Reddy DS. GABA-A receptor mediated antistress activity of neurosteroids in mice. Eur Neuropsychopharmacol., 1996; 6:77.

Kumar S, Nei M, Dudley J, Tamura K. MEGA: a biologistcentric software for evolutionary analysis of DNA and protein sequences.
Brief in Bioinfo., 2008; 9:299-306.

Kumar S, Stecher G Tamura K. MEGA7: Molecular Evolutionary Genetics Analysis version 7.0 for bigger datasets. Mol Biol Evol., 2016; $33: 1870-1874$

Lakshmi K V, Karadi RV, MallikarjunaRaoK, Gaviraj EN, Shinde GS, Studies on anti-stress activity of seeds aqueous extract of cuminumcyminumlinn, Int J Ayur Pharm Res., 2011; 2: 41-44.

Lotankar AR, Wankhede S, Sharma JB, and Momin A. AntiStress Activity of Flavonoids Rutin and Quercetin Isolated from the Leaves of Ficusbenghalensis. Int J Pharma Pharma Res., 2016; 5: 5-19.

Luján R, Shigemoto R, and López-Bendito G. Glutamate and GABA receptor signalling in the developing brain. Neuroscience, 2005; 130: 567-580.

Lee M, Kim Y, Lee B, Kwon S, Moon W, Hong K, Song Y, Morita K, Hahm D, Shim I and Her S. Novel antidepressant-like activity of caffeic acid phenethyl ester is mediated by enhanced glucocorticoid receptor function in the hippocampus. Evid Based Complement Alternat Med., 2014; 2014:1-10.

Mihic SJ and Harris R. GABA and the GABAA receptor. Alcohol Health Res. World., 1997; 21:127-131.

Miller PS, Aricescu AR. Crystal structure of a human GABAA receptor. Nature., 2014; 512:270-5.

Nutt D. GABA A Receptors : Subtypes, Regional Distribution and Function. J. Clin. Sleep Med., 2006;2: S7-S11.

Trott O, OlsonA J. AutoDockVina: improving the speed and accuracy of docking with a new scoring function, efficient optimization and multithreading. J Comp Chem., 2010;31:455-461.

Pawar VS, Shivakumar H. A current status of adaptogens: natural remedy to stress. Asian Pac J Trop Dis, 2012; 1:S480-90.

Romero LM and Butler LK. Endocrinology of Stress. Int J Com Psychol., 2007;20:89-95.

Roozendaal MM, de Krudf HF, Reuling RJ, Threels A, Swarts JJ, Wiegant VM, Matthejj JA. Inhibition of the LH surge by restraint stress in cyclic rats: studies on the role of GABAA and GABAB receptors. Stress. $1997 ; 1: 241-8$

Sahley BJ. GABA : The Brain and How it Affects Our Behavior, 2006; Pain Stress Publ.

Seeliger D, Groot BL. Ligand docking and binding site analysis with PyMOL and Autodock/Vina. J Comput Aided Mol Des., 2010; 24:417-422.

T. Friedman, "The effect of rosmarinic acid on immunological and neurological systems: a basic science and clinical review", J Restorative Med., 2015; 4:50-59.

Uddin A, Shahinuzzaman, Rana S, and Yaakob Z. Study of chemical composition and medicinal properties of volatile oil from clove buds (eugeniacaryophyllus). Int J of Pharma Sci Res, 2017;8: 895-899.

Vladimir-Knežević S, Blažeković B, Kindl M, Vladić J, LowerNedza A, and Brantner A. Acetylcholinesterase Inhibitory, Antioxidant and Phytochemical Properties of Selected Medicinal Plants of the Lamiaceae Family. Molecules., 2014; 19: 767-782.

Zahradníková L, Schmidt S, Sekretár S, and Janáč L. Determination of the antioxidant activity of Ginkgo bilobaleaves extract. J. Food Nutr Res, 2007; 46:15-19.

How to cite this article:

Negi A, Singh P, Taneja N, Mani S. Molecular-Docking Study of Anti-Stress Natural Compounds Against GABAa Receptor Portends the Novel Approach to Stress Treatment. J App Pharm Sci, 2018; 8(12): 038-043. 